\title{
Experiencia española en el proyecto Go-Lab
}

\author{
Iratxe Menchaca Sierra \\ Olga Dziabenko \\ Javier García Zubía \\ Universidad de Deusto. España. \\ iratxe.mentxaka@deusto.es \\ olga.dziabenko@deusto.es \\ zubia@deusto.es
}

Recibido: 3/2/2019

Aceptado: 16/1/2020

Publicado: 20/7/2020

\section{Resumen}

El proyecto Go-Lab está financiado dentro del Programa FP7 de la Unión Europea y su principal objetivo es fomentar el área STEM en alumnos jóvenes preuniversitarios. Cuenta con una veintena de socios de más de 15 países y se articula en tres grandes ejes: pedagogía, tecnología y despliegue en centros educativos. Pedagógicamente se basa en el aprendizaje por indagación (Inquiry Based Learning [IBL]), aspecto que desarrolla utilizando laboratorios en línea, ya sean virtuales o remotos, para obtener el mayor efecto en profesores y alumnos de secundaria y bachiller. La aportación española ha sido la más relevante en lo que se refiere al despliegue del ecosistema Go-Lab en colegios, puesto que supone cerca de un $25 \%$ del total de profesores que lo desarrollan y lo implementan en sus centros de enseñanza. Go-Lab se ha extendido a un total de 281 escuelas españolas. Se ha observado que las aplicaciones de dicho ecosistema facilitan la experiencia necesaria a los alumnos, de manera que estos puedan ser protagonistas de su aprendizaje desde un rol creador e investigador, lo que promueve el acercamiento juvenil a las disciplinas STEM.

Palabras clave: laboratorios remotos y virtuales; Go-Lab; STEM; aprendizaje por indagación; experimentación

\section{Resum. Experiència espanyola en el projecte Go-Lab}

El projecte Go-Lab està finançat dins del Programa FP7 de la Unió Europea i el principal objectiu que persegueix és el de fomentar l'àrea STEM en alumnes joves preuniversitaris. En formen part una vintena de socis de més de 15 països i s'articula en tres grans eixos: pedagogia, tecnologia i desplegament en centres educatius. Pedagògicament es basa en l'aprenentatge per investigació (Inquiry Based Learning [IBL]), aspecte que es desenvolupa utilitzant laboratoris en línia, ja siguin virtuals o remots, per obtenir l'efecte més gran possible en professors i alumnes de secundària i de batxillerat. L'article descriu aquests tres aspectes, fent un èmfasi especial en el procés de desplegament a Espanya i en els resultats que s'hi han obtingut. La contribució espanyola ha estat la més rellevant en referència al desenvolupament de l'ecosistema Go-Lab a les escoles, ja que representa prop del 25\% del total de professors que el desenvolupen i que l'implementen als seus centres docents. Go-Lab s'ha estès a un total de 281 escoles espanyoles. S'ha observat que les aplicacions d'aquest ecosistema proporcionen l'experiència necessària als estudiants, perquè puguin 
ser protagonistes del seu aprenentatge des d'un paper creatiu i investigador, la qual cosa promou l'aproximació juvenil a les disciplines STEM.

Paraules clau: laboratoris remots i virtuals; Go-Lab; STEM; aprenentatge per investigació; experimentació

\section{Abstract. Spanish experience in the Go-Lab Project}

Go-Lab is a project funded by the European Union FP7 programme to promote STEM education in pre-university students. The project includes about 20 members from more than 15 different countries and is constructed around three central ideas: pedagogy, technology and deployment in schools. Pedagogically, the project is rooted in inquiry-based learning (IBL), which is developed using virtual or remote online laboratories to maximize the effect on secondary education teachers and students. This paper describes these three facets, putting special emphasis on the deployment process and the results achieved in Spain. The Spanish contribution has been the most relevant to the development of the Go-Lab ecosystem in schools, since it accounts for nearly $25 \%$ of the total number of teachers who develop and implement Go-Lab in their schools and involves a total of 281 schools in the country. It has been observed that the Go-Lab ecosystem apps provide students the necessary experience to be protagonists of their learning through a creative and research role that promotes the youth approach to STEM disciplines.

Keywords: remote and virtual labs; Go-Lab; STEM; inquiry-based learning; experimentation

\section{Sumario}
1. Introducción
5. Impacto y resultados
2. Proyecto Go-Lab
6. Conclusión
Referencias bibliográficas

3. Implementación de Go-Lab en España

4. Diseño e implementación de ILS

\section{Introducción}

Go-Lab es un proyecto financiado por la UE dentro del Programa FP7 durante cuatro años (de 2013 a 2016). Su objetivo principal es la mejora de la enseñanza STEM en las aulas desde la perspectiva de la indagación científica, utilizando como soporte laboratorios en línea y aplicaciones de apoyo al alumno y al profesor.

El proyecto contó con 18 socios de 11 países diferentes y fue coordinado por Ton de Jong (Universidad de Twente, Holanda). La Universidad de Deusto fue una de las dos instituciones españolas (junto con la UNED) que participó en el proyecto, y lo hizo tanto en la parte técnica como en la parte de comunidad de profesores, donde tuvo el rol de National Contact para España.

Desde el punto de vista pedagógico, la aportación más relevante del proyecto es el diseño de ILS (Inquiry Learning Spaces) por parte de los profesores. Tecnológicamente, los elementos más característicos de Go-Lab son el uso de laboratorios en línea (con una elevada complejidad tecnológica), el uso 
de aplicaciones de apoyo y de Graasp (<www.graasp.eu $>$ ) como herramienta de autoría para elaborar ILS. Estas ILS son publicadas por los profesores en un repositorio (<www.golabz.eu/>) y cualquiera de ellos puede utilizar una ILS creada por otro profesor, puede crear la suya propia desde cero o puede partir de lo realizado por otro profesor y adaptarlo. Todos los resultados están abiertos para la comunidad de profesores, sean o no miembros de Go-Lab.

El trabajo se estructura en cinco secciones adicionales. En la segunda se describe el proyecto detalladamente, para abordar su despliegue en España en la tercera sección. La siguiente se centra en las ILS, en sus partes y en su desarrollo tecnológico. Los resultados obtenidos en los cuatro años de Go-Lab en España se presentan en la quinta sección, que contiene las conclusiones y el trabajo futuro centrado en el nuevo proyecto, llamado Next-Lab.

\section{Proyecto Go-Lab}

Los alumnos aprenden de manera más eficiente cuando perciben la importancia de lo que estudian, cuando se involucran en ello y cuando se responsabilizan de los resultados finales de su educación. En este sentido, el proyecto Go-Lab fue originado para crear un marco pedagógico para el aprendizaje de la indagación con laboratorios en línea que permitiera a los estudiantes aprender nuevas cosas descubriéndolas a través de la aplicación del método científico. El proyecto transcurrió entre 2012 y 2016 y fue financiado por el Programa FP7.

El objetivo principal de Go-Lab era animar a los jóvenes a participar en temas de ciencia, adquirir conocimientos científicos y experimentar la cultura de practicar ciencia básica y ciencia aplicada de alto nivel.

El portal Go-Lab se creó también con el objetivo de generar una federación de laboratorios remotos y laboratorios virtuales, además de formar a los docentes para capacitarlos a la hora de integrar pedagógicamente estos laboratorios en los entornos de aprendizaje apoyándose en herramientas para tal fin, como son las aplicaciones de Go-Lab.

La infraestructura de Go-Lab proporciona un acceso fácil e intuitivo a los repositorios de laboratorios remotos y laboratorios virtuales. Para ello, Go-Lab proporciona:

- Acceso y uso de instrumentos científicos, como laboratorios remotos y virtuales.

- Acceso a datos y archivos de investigación.

- Uso de aplicaciones modernas para la adquisición, el análisis y la visualización de datos.

- Espacios de aprendizaje estructurados, basados en un ciclo de investigación bien definido.

- Aplicaciones de apoyo para el proceso de investigación.

- Aplicaciones de apoyo para la comunicación y la colaboración en el estudio científico. 
El ecosistema de Go-Lab ya se utiliza en 30 países europeos, así como en Estados Unidos, India, Rusia, Colombia, Brasil, Turquía, Canadá, Irán, Indonesia, Australia, etc. El ecosistema está disponible en 27 idiomas. Actualmente contamos con un total de casi 10.000 usuarios registrados en la plataforma de diseño de Go-Lab y 15.000 visitas mensuales. Cerca de 500 laboratorios en línea están disponibles para el uso de los profesores. Además, hay 42 aplicaciones de Go-Lab que apoyan la investigación, el aprendizaje analítico, la comunicación y los métodos de colaboración.

\subsection{Aprendizaje basado en la indagación}

En la actualidad existe un amplio consenso en torno a los enfoques basados en la investigación o en la indagación. La investigación activa y la experimentación por parte de los alumnos son necesarias para motivarlos hacia el estudio de las áreas científicas y de la ciencia en sí misma (Bybee, 2002; De Jong, Linn y Zacharia, 2013). De hecho, varios autores defienden que la investigación debe ser parte del plan de estudios de los estudiantes y por ello es fundamental el desarrollo de competencias relacionadas con la resolución de problemas, la discusión, el razonamiento, la argumentación y el análisis, habilidades estrechamente ligadas al método científico (National Coalition for Literacy, 2010; Rocard et al., 2007; EACEA P9 Eurydice, 2011).

La indagación es el método de aprendizaje mediante el cual se enseña a los estudiantes a ser investigadores. Los alumnos están motivados e involucrados a la hora de hacer preguntas científicas, de realizar experimentos activos, de formular conclusiones a partir de pruebas, de evaluar sus explicaciones y de comunicar y justificar sus argumentos propuestos. Existen evidencias científicas que demuestran que la investigación conduce a una mejor adquisición del conocimiento y del dominio (De Jong, 2006). En entornos de aprendizaje por indagación destacan las interrelaciones entre el pensamiento y la acción, en contraste con la educación científica tradicional, que pone más énfasis en aspectos conceptuales y racionales de la investigación (Paavola y Hakkarainen, 2018). Un metaanálisis de 2010 que analizó 138 estudios indicó una clara ventaja en las prácticas didácticas basadas en la investigación sobre otras formas de instrucción en relación con la comprensión conceptual que los estudiantes obtienen de su experiencia de aprendizaje (Minner, Levy y Century, 2010).

A través de Go-Lab los estudiantes utilizan la estructura y las fases del método científico gracias a las aplicaciones. Dichas aplicaciones orientan y guían el proceso hacia la obtención de resultados y de conclusiones, reflexionando sobre los datos y la observación de los experimentos. Los maestros pueden usar este enfoque en la didáctica de sus clases como espacios de aprendizaje en línea o de actividades no conectadas. 


\subsection{Laboratorios remotos y virtuales para el aprendizaje de la investigación}

Los laboratorios reales se utilizan en el campo de la educación por multitud de razones. Según Hofstein y Lunetta (2004), los experimentos científicos reales de laboratorio ayudan a comprender los conceptos científicos y suscitan interés y motivación entre el alumnado. Balamuralithara y Woods (2009) hablaron sobre los procedimientos de seguridad, así como sobre aprender a utilizar los sentidos humanos para las observaciones. Feisel y Rosa (2005) incluyeron además el aprendizaje a partir de los errores y del aprendizaje en equipo como beneficios de los laboratorios prácticos.

Sin embargo, varios estudios que se enfocaron explícitamente en el uso de manipulaciones físicas (Chambers, Chuen Yee Lo y Allen, 2008) y en la comparación entre virtuales y reales no encontraron ventajas en el componente físico de los experimentos (Corter, Esche, Chassapis, Ma y Nickerson, 2011; Zacharia y Olympiou, 2011).

Las comparaciones entre experimentos simulados y reales demostraron que ambos enfoques podían resultar igual de eficaces en la adquisición del conocimiento. Sin embargo, sí hay algunos trabajos que han evidenciado ventajas de los laboratorios virtuales sobre los reales, por ejemplo: Huppert, Blasius y Stone (2002, microbiología), Finkelstein, et al. (2005, circuitos eléctricos) y Bell y Trundle (2008, fases lunares).

Los laboratorios educativos manipulados a distancia («laboratorios remotos») proporcionan a los estudiantes la oportunidad de recopilar datos sobre una instalación física real de laboratorio y de equipos reales desde lugares remotos. Como alternativa hay laboratorios virtuales que simulan el equipo real.

Los laboratorios remotos tienen ventajas significativas para el aprendizaje y ofrecen una visión realista de la práctica científica, incluyendo aspectos prácticos como el mantenimiento y la creación del experimento, errores experimentales de medición, etc.

Los laboratorios virtuales tienen ventajas propias. Lo más significativo de ellos es que los estudiantes pueden experimentar repetidamente. No se requieren requisitos técnicos especiales excepto el dispositivo conectado a Internet. Por lo tanto, las ideas de aprendizaje pueden ser rápidamente probadas y evaluadas. Otra ventaja de los laboratorios virtuales es que los procesos experimentales pueden simplificarse, destacando las variables esenciales para el proceso de aprendizaje.

En conclusión, aunque los laboratorios remotos y los virtuales tienen un valor específico desde un punto de vista educativo, ambos pueden utilizarse en un mismo escenario de aprendizaje y cada uno de ellos tener sus propios objetivos de aprendizaje (Ma y Nickerson, 2006; Dziabenko et al., 2019).

El experimento puede ser considerablemente mejorado por la combinación de los dos mundos, el remoto y el virtual, mediante la mezcla de ellos. En Go-Lab existen diferentes formas de combinar los dos tipos de experimentación. Para ello, Go-Lab ha creado una red o federación de laboratorios que proporcionan acceso a un gran conjunto de laboratorios científicos remotos, 
como las infraestructuras del CERN (LHC, ATLAS, CMS); la Red Global de Telescopios del Observatorio Las Cumbres, en Hawai o Australia; La misión Gaia, de la ESA, y las infraestructuras de algunas universidades de ciencia y tecnología.

\section{Implementación de Go-Lab en España}

El impacto de Go-Lab en España se debe principalmente a una estrategia de implementación que se ha llevado a cabo desde el inicio del proyecto. En dicha estrategia se han previsto los canales de difusión necesarios para llegar a un elevado número de centros y de profesores, se han previsto mecanismos e infraestructuras para soportar la comunicación con dichos agentes y se han planificado diferentes acciones de formación y acompañamiento para asegurar la permanencia de los agentes en el proyecto.

A continuación se presentan las acciones llevadas a cabo para alcanzar los indicadores de impacto obtenidos en España para el proyecto Go-Lab.

\subsection{Captación}

El primer objetivo que se ha propuesto el equipo de Go-Lab en España desde la Universidad de Deusto es dar a conocer el proyecto al mayor número de profesores posible en toda la geografía española. Este primer paso busca captar la atención de una cantidad elevada de docentes con el fin de que ellos mismos busquen los canales específicos para conocer aún más el proyecto y recibir la formación necesaria acerca de él. Para ello se ha presentado el proyecto en varios congresos y conferencias de ámbito educativo y tecnológico a nivel español y se ha establecido contacto con las administraciones educativas de diferentes comunidades autonómicas, así como con diferentes redes de centros y fundaciones educativas.

\subsection{Formación}

La formación es la estrategia fundamental para que el proyecto Go-Lab y todos los recursos que este ofrece se implementen realmente en los centros educativos de España. Gracias a la formación se dota al profesorado de las habilidades y de los mecanismos necesarios para utilizar tanto los laboratorios remotos y virtuales como las ILS o los recursos educativos generados en el proyecto. La formación se ha llevado a cabo en diferentes momentos a lo largo del proyecto y se ha dirigido a distintos destinatarios. A nivel general, se han ofrecido trimestralmente varios seminarios de formación en línea a todos los inscritos en el formulario inicial de Go-Lab. Además, para determinados grupos de profesores centralizados en algunas áreas geográficas, como por ejemplo Galicia, Cataluña o el País Vasco, se han proporcionado estas mismas formaciones a nivel presencial. 


\subsection{Difusión y comunicación}

Para garantizar la permanencia del profesorado en los procesos de formación y para mantener su interés y continuidad en Go-Lab, se ha diseñado un portal específico para toda la actividad del profesorado de Go-Lab. El portal <http:// Go-Labspain.deusto.es/> es la infraestructura necesaria para mantener continuamente informada a la comunidad educativa. En este espacio se comparten noticias referentes a las novedades en el proyecto, a la actualización de las aplicaciones, a la publicación de nuevas ILS que pueden ser de interés general, etc. Además, es un espacio ideal para compartir ejemplos de buenas prácticas, para compartir fotos, vídeos o testimonios de alumnos y profesores. Otro espacio decisivo dentro de la web ha sido el mapa, donde se da visibilidad a todos los centros participantes en Go-Lab y se facilita información sobre el estado en el cual se encuentran dichos centros, es decir, si han recibido la formación, si han implementado el programa en sus aulas, etc.

\subsection{Seguimiento}

Por último, una labor decisiva es el acompañamiento al profesorado. En cualquier proceso de formación y de innovación educativa los docentes necesitan sentirse acompañados para solventar los obstáculos que aparecen tanto en la fase de diseño como en la de implementación en el aula. Desde el equipo de Go-Lab en España se ha realizado un seguimiento continuo del profesorado que ha participado en los procesos de formación. En dicho seguimiento se le ha facilitado asesoramiento sobre el uso y la comprensión de los laboratorios, sobre el diseño de las ILS, sobre la selección de aplicaciones, sobre las pautas de utilización en el aula, etc. Es importante que el profesorado perciba que la plataforma y la tecnología que está utilizando es consistente, que no le va a dar fallos durante la implementación y que, en el caso de que exista cualquier fallo, va a haber alguien que le facilite información para solucionarlo.

\section{Diseño e implementación de ILS}

El objetivo específico de Go-Lab, el output del proyecto, es el diseño y uso de ILS. Se describe una ILS como una combinación inteligente de un experimento científico, con un laboratorio en línea y con varias aplicaciones de apoyo y soporte a la experimentación bajo un enfoque pedagógico inspirado en el aprendizaje por indagación. Una ILS es un ejemplo de entorno activo y colaborativo de aprendizaje en el cual se presta especial atención al desarrollo de competencias transversales (Eizaguirre, Altuna y Fernández, 2018). En la figura 1 se describen los aspectos más significativos de una ILS.

Desde el punto de vista pedagógico, y viendo la imagen anterior, una ILS se compone de cinco fases consecutivas (aunque se puede ir hacia atrás y hacia delante) (Pedaste et al., 2015): 
Figura 1. Fases de una ILS: orientación, conceptualización, experimentación, conclusiones y discusión

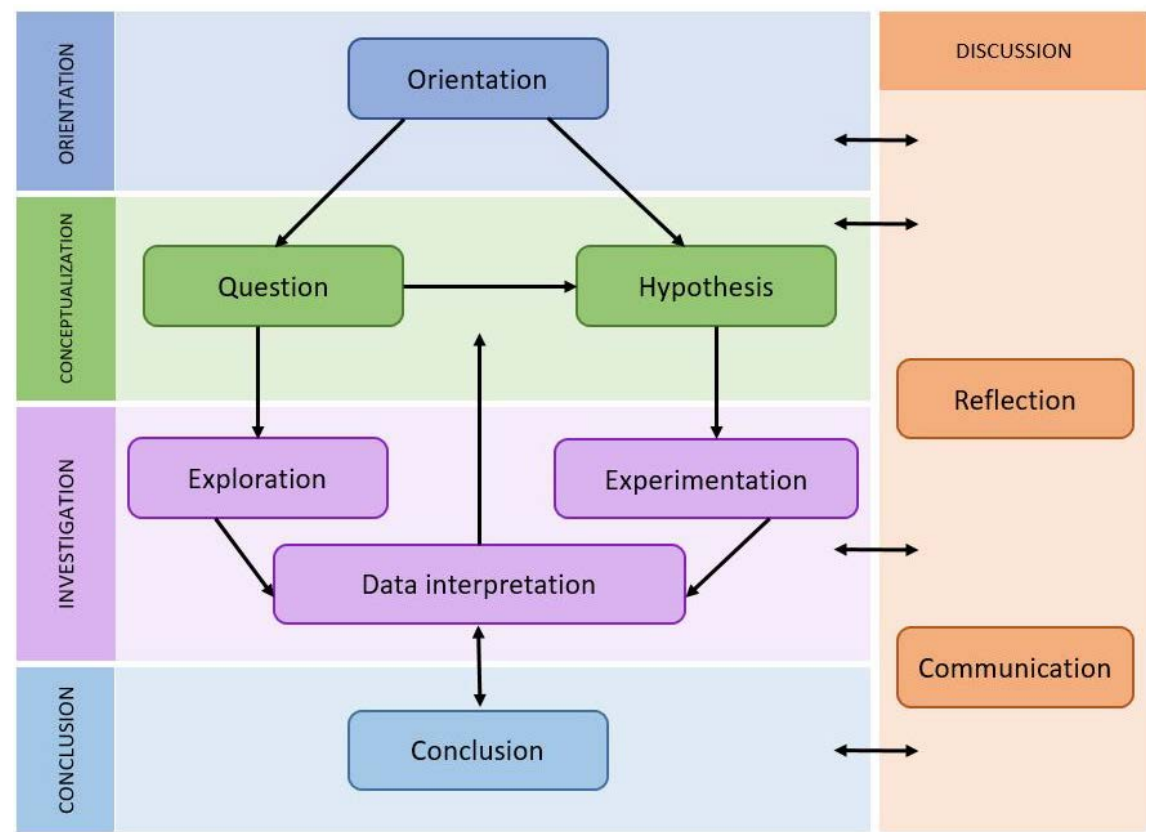

Fuente: Pedaste et al. (2015).

- Fase de orientación. El objetivo de esta primera fase es capturar la atención del alumno mediante la observación de un hecho sorprendente o estimulante desde el punto de vista científico.

- Fase de conceptualización. Esta fase debe acabar con la elaboración de una lista de hipótesis (cuatro o cinco, por ejemplo) por parte del alumno. Debe aportar al alumno la información suficiente para conocer las variables y establecer posibles hipótesis entre ellas.

- Fase de experimentación. El elemento principal de esta fase es el laboratorio en línea (virtual o remoto), que permite realizar el experimento o los experimentos que combinan las variables de las hipótesis planteadas en la fase anterior. Una vez hecho el experimento es crucial que el alumno recoja los datos, bien en forma de número o bien en forma de gráfica, para poder analizarlos en la siguiente fase. La indagación se desarrolla en experimentos.

- Fase de conclusiones. Una vez realizados los experimentos que contrastan las hipótesis, queda determinar cuáles son ciertas y cuáles no a la vista de los resultados obtenidos. Es muy importante que el profesor explique a los alumnos que una buena hipótesis no es aquella que es cierta, sino que lo importante es que sea verificable mediante un experimento. La indagación ofrece certezas al alumno. 
- Fase de discusión. Esta es una fase abierta. En ella el profesor y los alumnos toman la experiencia científica como excusa para llevar a cabo un debate de mayor calado, quizá la ecología, la sostenibilidad, los hechos alternativos o, por ejemplo, introducir el tema de la siguiente ILS. La indagación favorece el pensamiento crítico en el alumno.

Una vez introducido el término ILS pasamos a profundizar en los laboratorios. Para empezar, un laboratorio en línea se distingue porque solo es accesible mediante Internet, y además puede ser virtual o remoto. En un laboratorio virtual nada es real, todo es simulado, aunque muestra una gran verosimilitud. A veces se distingue entre simulador y laboratorio virtual según lo parecido que sea la interfaz al usado en el laboratorio clásico. Un ejemplo típico de laboratorio virtual son las simulaciones PhET, y un caso extremo de realismo se puede encontrar en <www.labster.com>.

En un laboratorio remoto el experimento es real y por tanto todos los datos lo son también. En este caso el control del experimento se hace vía Internet. Este tipo de laboratorios son más relevantes, aunque no son suficientemente populares entre el profesorado y los colegios. Muchos centros de investigación ofrecen laboratorios remotos (<http://relle.ufsc.br/>, <www.weblab.deusto. es> etc.).

La imagen (figura 2) muestra un ejemplo del experimento de Arquímedes reproducido en el laboratorio virtual Splash: Virtual Buoyancy Laboratory. La figura 3 muestra el mismo experimento, pero con un laboratorio remoto de WebLab-Deusto (Orduña et al., 2018).

Go-Lab ofrece en su portal para los diseñadores cerca de 400 laboratorios en línea clasificados según su temática, tipología (virtual y remoto), lenguaje, etc. De esta forma el profesor puede optar por el laboratorio que más se ajuste a sus necesidades o seguir las recomendaciones de un colega de proyecto. Además, Go-Lab ofrece unas 40 aplicaciones, la mayoría de las cuales han sido diseñadas exprofeso para el proyecto. A continuación, se describen tres aplicaciones fundamentales con el objetivo de ilustrar al lector con el resto de aplicaciones. 
Figura 2. Ejemplo de laboratorio virtual (Splash)

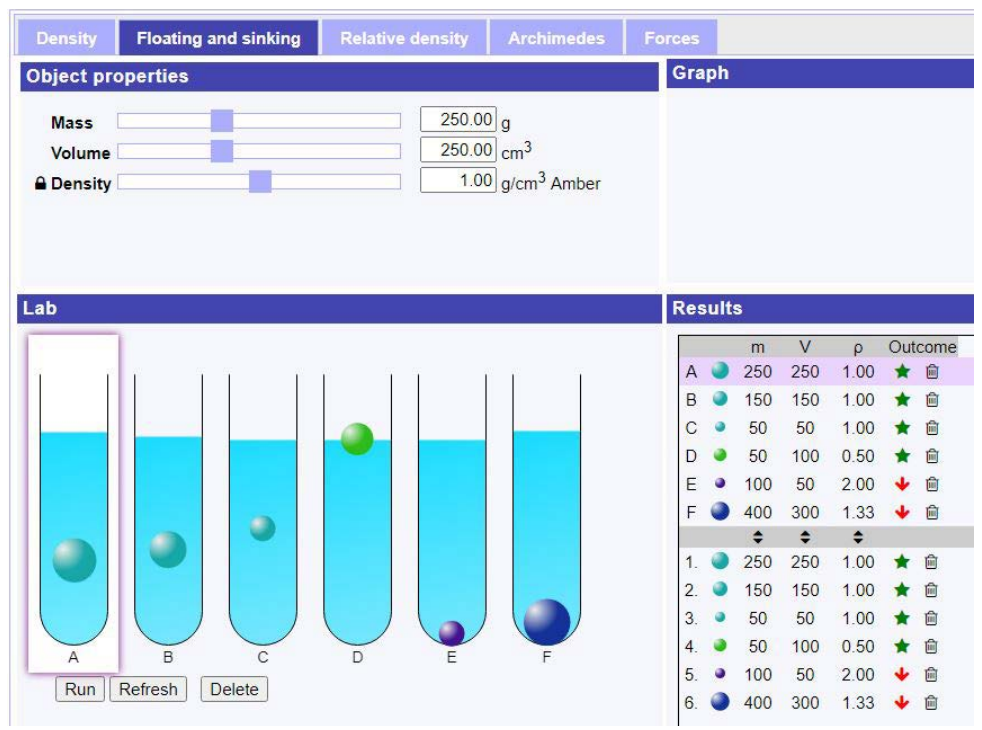

Fuente: Splash: Virtual Buoyancy Laboratory disponible en el repositorio Go-Lab (<http://www.golabz.eu/>).

Figura 3. Ejemplo de laboratorio remoto (WebLab-Deusto)
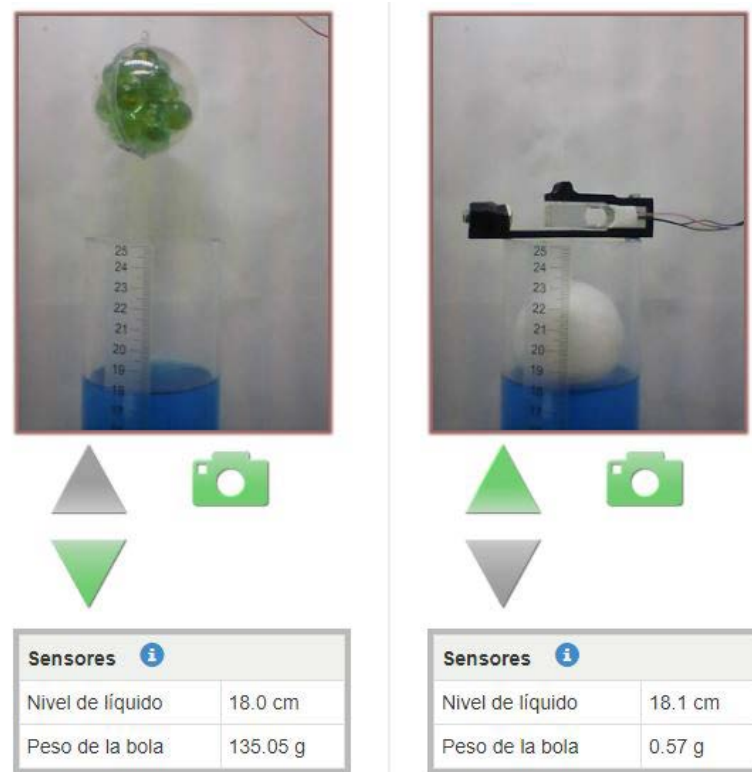

\begin{tabular}{|l|l|}
\hline Sensores & \\
\hline Nivel de liquido & $18.1 \mathrm{~cm}$ \\
\hline Peso de la bola & $0.57 \mathrm{~g}$ \\
\hline
\end{tabular}

Fuente: Orduña et al. (2018). 
Hypothesis tool. Cuando el profesor incluye esta aplicación en la ILS ofrece al alumno la posibilidad de escribir hipótesis arrastrando y combinando partes de hipótesis ya preparadas por el profesor. El profesor puede (y debe) configurar la aplicación según sus necesidades.

La imagen muestra la hypothesis tool (figura 4) para el principio de Arquímedes. El alumno añade hipótesis y puede indicar cuán seguro está de la validez de una hipótesis. Todo ese trabajo queda almacenado en la propia página web, siempre disponible para el profesor.

Figura 4. Hypothesis tool

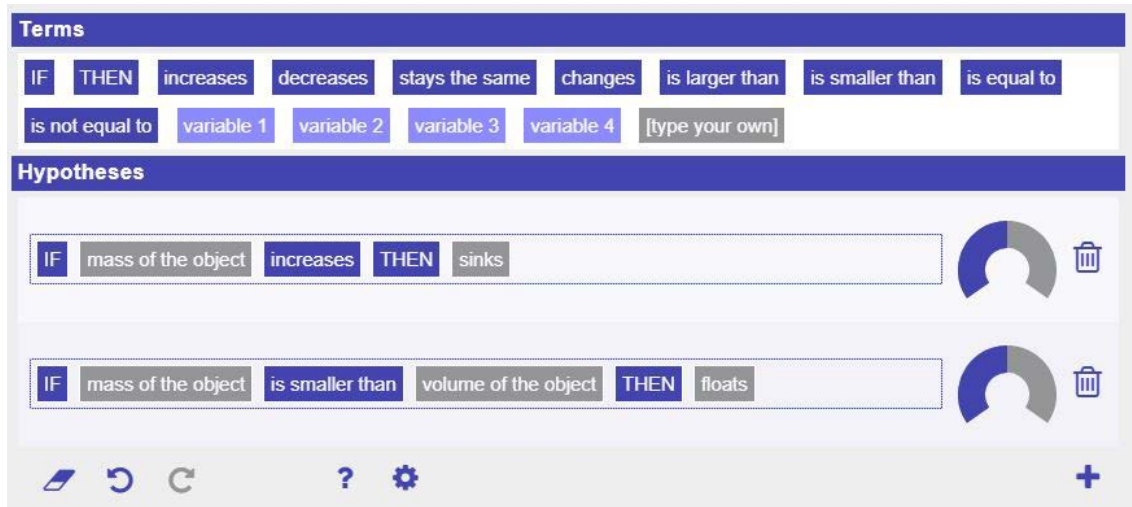

Fuente: Hypothesis tool disponible en el repositorio Go-Lab (<http://www.golabz.eu/>).

Observation tool. Cada vez que el alumno completa un experimento, él debe tomar nota del resultado, bien como un número en una tabla o mediante una observación escrita que quedará registrada. En la imagen (figura 5) se ve la observación añadida por un alumno e indica si un objeto ha flotado o no, su volumen y su masa.

Figura 5. Observation tool

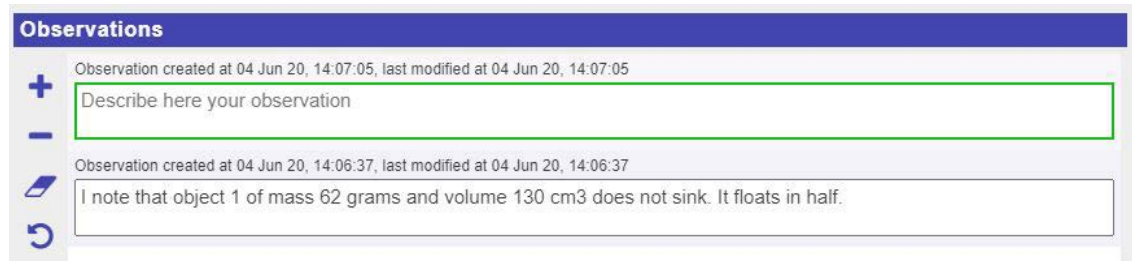

Fuente: Hypothesis tool disponible en el repositorio Go-Lab (<http://www.golabz.eu/>). 
Conclusion tool. Finalmente el alumno debe demostrar al profesor su capacidad de análisis para decidir si una hipótesis es cierta o no y en base a qué datos u observaciones lo considera así, pero en vez de darle al profesor una serie de notas sueltas más o menos elaboradas, la herramienta le ofrece la posibilidad de conjugar los tres aspectos haciendo drag and drop. Para el alumno es fácil y le enseña a ser sistemático, y al profesor le facilita la corrección. En este caso el alumno elige una hipótesis, decide si es cierta o no y le asocia una observación o un gráfico. La imagen da una idea del proceso.

Figura 6. Conclusion tool

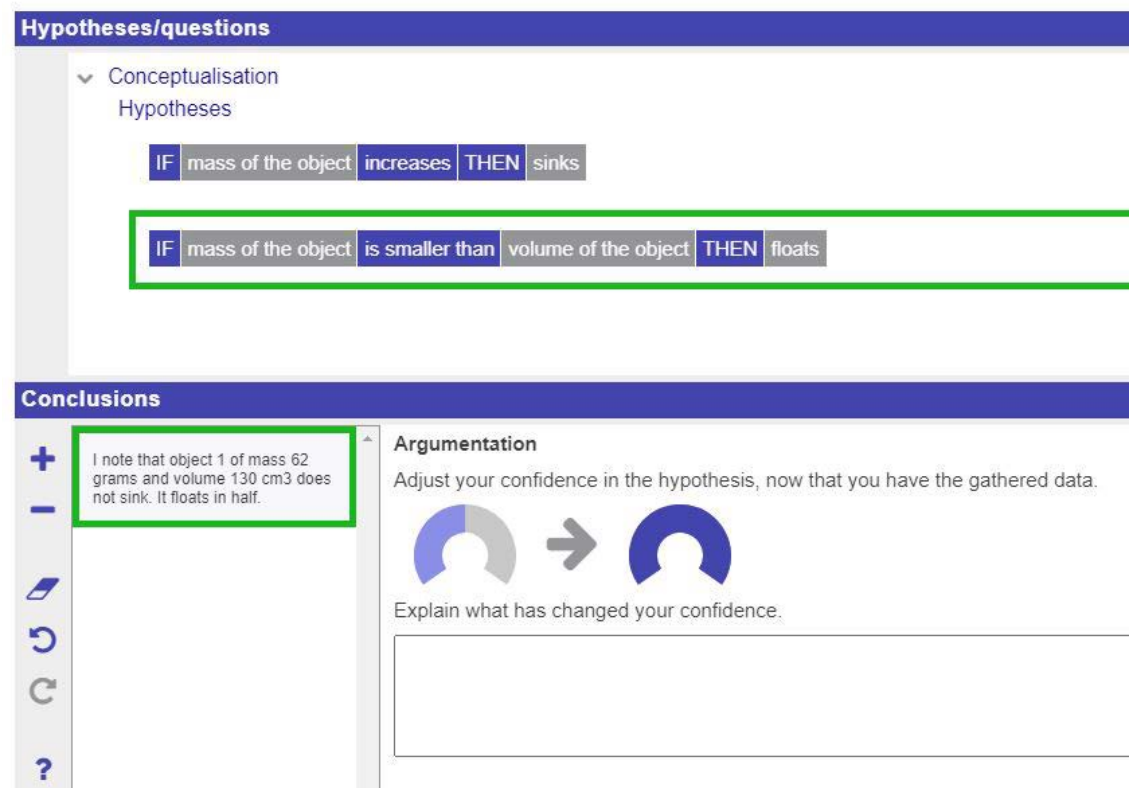

Fuente: Conclusion tool disponible en el repositorio Go-Lab (<http://www.golabz.eu/>).

Existen más aplicaciones, algunas de ellas claramente reconocibles, otras interesantes y un último grupo con algunas más específicas. Por ejemplo: hay una aplicación para obtener mapas conceptuales, para diseñar un experimento, para subir ficheros o para crear test y cuestionarios; también hay aplicaciones que permiten saber qué, cuándo y cómo está haciendo el alumno (Learning Analytics, LA). Utilizando estas aplicaciones (cerca de 15 de un total de 40), el profesor puede saber cuándo entró un determinado alumno en la ILS, qué resultados obtuvo y en qué orden. Las aplicaciones de LA suelen ser muy interesantes para el profesor, tanto en su relación con el alumno como con el centro escolar. 
Queda por último saber cómo se crea una ILS: usando una herramienta llamada Graasp. A la hora de diseñar una ILS, un profesor puede seguir tres caminos:

— Va al repositorio denominado <www.golabz.eu/> y busca qué ILS se adapta a lo que él necesita. Si lo encuentra, lo copia y lo usa. Este trabajo puede llevar no más de 5 minutos.

- Va al repositorio, busca y encuentra una ILS que se adapta a sus necesidades, pero ve que es mejorable (con un nuevo vídeo, con un laboratorio distinto, etc.), así que copia la ILS, la modifica y la publica como nueva. Este trabajo no debería llevarle más de 3 horas, ya que la ILS ya está creada.

- Va al repositorio, busca una ILS pero no la encuentra, y decide crear una ILS desde cero. Este trabajo puede llevarle entre 5 y 10 horas, según su experiencia y según la complejidad de la ILS a diseñar.

\section{Impacto y resultados}

En primer lugar, es relevante analizar el número de profesores y de centros educativos de toda España que han participado en las diferentes fases del proyecto Go-Lab. A continuación, se muestra una tabla con el número de centros y de profesores implicados en la fase de difusión, en la fase de formación y en la fase de diseño e implementación (tabla 1).

Tabla 1. Muestra de profesores y estudiantes en las distintas fases

\begin{tabular}{lcc}
\hline & Núm. profesores & Núm. centros \\
\hline Difusión & 405 & 281 \\
Formación & 178 & 116 \\
Diseño e implementación & 40 & 38 \\
\hline
\end{tabular}

Fuente: elaboración propia.

Aún más importante que el número de profesores a los que ha llegado Go-Lab en España (405), es el número de centros. Acceder a 281 centros educativos de la geografía española implica la posibilidad de multiplicar aún más el impacto del proyecto a nivel nacional.

Igualmente, se pueden analizar los datos referentes a las ILS diseñadas en España, en español, en catalán, en euskera y en gallego. Dichas ILS han sido diseñadas por el equipo del proyecto Go-Lab en España y por el conjunto de profesores que han participado en la fase de diseño e implementación (tabla 2). 
Tabla 2. Idioma de las ILS publicadas

\begin{tabular}{lc}
\hline & ILS publicadas \\
\hline Español & 55 \\
Catalán & 7 \\
\hline Euskera & 16 \\
Gallego & 17 \\
\hline Inglés & 156 \\
Otros idiomas (18) & 330 \\
\hline Total & 581 \\
\hline
\end{tabular}

Fuente: elaboración propia.

Si observamos los datos, encontramos que 95 ILS han sido diseñadas en lenguas locales. Si comparamos esta cifra con el total de ILS creadas (581), observamos que un 16\% de los recursos educativos de Go-Lab han sido diseñados por la comunidad de profesores de Go-Lab en España. Además, a este número de ILS habría que añadir un porcentaje importante de las ILS que han sido diseñadas en inglés, puesto que varios profesores de nuestra comunidad han diseñado sus ILS en inglés.

Otro dato importante que nos permite apreciar el trabajo realizado por la comunidad de Go-Lab en España es la comparación entre el número de ILS en lenguas locales (95) y el número de ILS diseñadas en el resto de idiomas, excluyendo el inglés. Si consideramos que hay 18 lenguas diferentes al inglés y a las nuestras locales, observamos que la media de ILS por idioma es de 18,3. Comparando este número de ILS por idioma con el 95 que hemos obtenido a nivel nacional, podemos apreciar el esfuerzo y la implicación de la comunidad de Go-Lab en España.

Entre el profesorado que ha recibido formación sobre Go-Lab disponemos de datos de 59 docentes que han completado todo el proceso de la formación, es decir, se han formado en el uso de laboratorios y de ILS, así como en el diseño de ILS. A continuación mostramos los datos sobre el uso que estos profesores han hecho finalmente de Go-Lab. Un 78\% de los profesores que se han formado en Go-Lab han llegado a diseñar sus propias ILS (tabla 3). 
Tabla 3. Resultados sobre el uso de las ILS

\begin{tabular}{llcccc}
\hline & Frecuencia & Porcentaje & $\begin{array}{c}\text { Porcentaje } \\
\text { válido }\end{array}$ & $\begin{array}{c}\text { Porcentaje } \\
\text { acumulado }\end{array}$ \\
\hline \multirow{3}{*}{ Válido } & $\begin{array}{l}\text { Búsqueda de } \\
\text { laboratorios }\end{array}$ & 6 & 10,2 & 10,2 & 10,2 \\
& $\begin{array}{l}\text { Uso de ILS } \\
\text { existentes }\end{array}$ & 7 & 11,9 & 11,9 & 22 \\
& Diseño de ILS & 46 & 78 & 78 & 100 \\
& Total & 59 & 100 & 100 & \\
\hline
\end{tabular}

Fuente: elaboración propia.

A continuación se muestran datos acerca de cómo los profesores valoran las aportaciones de Go-Lab en su práctica docente, es decir, si consideran que su uso aumenta la utilización de experiencias de laboratorios en el aula, si cumple con sus expectativas como docentes y cubre sus necesidades de enseñanza y si la comunidad de Go-Lab le permite conocer ejemplos de buenas prácticas para incorporarlas en su docencia. En este sentido, en una escala del $1 \mathrm{al}$ 7, de menor a mayor grado de conformidad con las afirmaciones, el profesorado valora positivamente las contribuciones de Go-Lab a su práctica docente.

Tabla 4. Resultados de la valoración del profesorado en relación con el uso de Go-Lab en el aula

\begin{tabular}{lccc}
\hline & N & Media & $\begin{array}{c}\text { Desviación } \\
\text { estándar }\end{array}$ \\
\hline Las herramientas de Go-Lab apoyan mi práctica docente & 59 & 4,95 & 1,074 \\
\hline Me ayuda a integrar el trabajo de laboratorio en mis clases & 58 & 4,00 & 1,451 \\
$\begin{array}{l}\text { Las capacidades del portal Go-Lab cumplen mis expec- } \\
\text { tativas }\end{array}$ & 57 & 4,88 & 1,255 \\
\hline $\begin{array}{l}\text { Las ILS existentes apoyan mi práctica docente } \\
\text { El intercambio de experiencias mejora mi práctica con }\end{array}$ & 52 & 4,69 & 1,515 \\
Go-Lab & 28 & 5,71 & 1,013 \\
\hline
\end{tabular}

Fuente: elaboración propia.

Igualmente, podemos observar las valoraciones del profesorado en relación con la usabilidad de la plataforma Go-Lab y de sus ILS. En una escala del 1 al 7, de menor a mayor grado de conformidad con las afirmaciones, el profesorado sí ha expresado unos valores de frustración con respecto al uso de la plataforma. Estos valores se deben principalmente a que, en diferentes fases de la formación, la plataforma ha sido sometida a diversas actualizaciones de mejora. 
Tabla 5. Resultados de la valoración del profesorado en relación con la usabilidad de la plataforma Go-Lab

\begin{tabular}{lccc}
\hline & N & Media & $\begin{array}{c}\text { Desviación } \\
\text { estándar }\end{array}$ \\
\hline Utilizar el portal Go-Lab es una experiencia frustrante & 57 & 2,60 & 1,412 \\
El portal Go-Lab es fácil de usar & 57 & 4,72 & 1,250 \\
\hline Requiere demasiado tiempo trabajando con el portal Go-Lab & 57 & 3,89 & 1,578 \\
\hline
\end{tabular}

Fuente: elaboración propia.

En relación con la intención del profesorado de implementar Go-Lab en el futuro o de compartir los recursos creados con otros grupos de profesorado, en una escala del $1 \mathrm{al} \mathrm{7,} \mathrm{de} \mathrm{menor} \mathrm{a} \mathrm{mayor} \mathrm{grado} \mathrm{de} \mathrm{conformidad} \mathrm{con} \mathrm{las} \mathrm{afirma-}$ ciones, un importante número de profesores asegura estar de acuerdo con compartir los recursos y con continuar utilizando Graasp para el diseño de ILS.

Tabla 6. Resultados sobre la intención del profesorado de utilizar Go-Lab en el aula

\begin{tabular}{lccc}
\hline & $\boldsymbol{N}$ & Media & $\begin{array}{c}\text { Desviación } \\
\text { estándar }\end{array}$ \\
\hline $\begin{array}{l}\text { Me gustaría compartir ILS con otros profesores que usen } \\
\text { Go-Lab }\end{array}$ & 41 & 6,39 & 0,666 \\
$\begin{array}{l}\text { Creo que puedo usar Graasp de forma sistemática para } \\
\text { crear ILS }\end{array}$ & 50 & 5,24 & 1,437 \\
\hline
\end{tabular}

Fuente: elaboración propia.

Los aspectos positivos que destacan los profesores sobre Go-Lab y sus recursos son la facilidad de uso de las aplicaciones que complementan las ILS, la sencillez y claridad en la distribución de la información en la plataforma, el acceso a un gran repositorio de laboratorios y la utilización de aplicaciones para orientar el método científico. Entre los aspectos negativos se han identificado la complejidad de algunos laboratorios, el sistema de búsqueda y filtrado de laboratorios o ILS y la falta de estabilidad en determinadas ocasiones provocada por las actualizaciones. Además, el profesorado cree que pueden darse algunos obstáculos a la hora de implementar Go-Lab en el aula, por ejemplo: las habituales dificultades técnicas de conexión de los equipos, el sistema de colas para la espera en la gestión del uso de los laboratorios remotos o la propia barrera del idioma.

Por último, una cuestión fundamental es la reflexión acerca de los cambios pedagógicos que se tienen que dar para aumentar la efectividad de Go-Lab en el aula. En este sentido, el profesorado considera que este enfoque metodológico basado en la indagación requiere mayores niveles de autonomía del alumno, y este aún no está acostumbrado a utilizar modelos menos guiados. Es un proceso que debe ir modificándose poco a poco hacia mayores niveles de autonomía y autoaprendizaje del educando. Una necesidad detectada también por el profesorado son los sistemas de evaluación, retroalimentación y segui- 
miento del trabajo estudiantil. Estas funcionalidades se han incorporado ya hoy en día dentro del catálogo de aplicaciones de Go-Lab.

\section{Conclusión}

En los cuatro últimos años, el ecosistema Go-Lab (Go-Lab y su continuación Next-Lab) es una marca de calidad pedagógica y tecnológica en los colegios en España. Actualmente cuenta con cerca de 500 laboratorios en línea, un número similar de ILS y más de 40 aplicaciones de apoyo al aprendizaje, enseñanza y evaluación. En este desarrollo la comunidad española de Go-Lab supone cerca de un $25 \%$ del total de profesores que lo desarrollaron y lo implementaron en sus centros de enseñanza y en sus clases. Más del 30\% de los recursos educativos diseñados proviene de profesores españoles. En su conjunto la aportación española ha sido la más relevante al despliegue del ecosistema Go-Lab en colegios.

Go-Lab ha impactado de forma clara en el desarrollo curricular docente, y su bandera es el desarrollo de actividades de indagación (ILS) para alumnos y profesores, teniendo como centro sus propias experiencias científicas y de laboratorio. La incorporación de los laboratorios remotos a los virtuales y su mayor realismo aportan a Go-Lab y a sus protagonistas elementos prácticos en el aula y reflexión docente. Se ha observado que las aplicaciones como Hypothesis, Questions Scratchpad, Conclusion, Data View y Report Form facilitan la experiencia a los alumnos, de manera que estos puedan ser protagonistas de su aprendizaje desde un rol creador e investigador, lo que promueve el acercamiento juvenil a las disciplinas STEM.

El primer eslabón del ecosistema Go-Lab ha sido el proyecto de este nombre, y el segundo es el proyecto Next-Lab, aprobado por Horizon 2020: The Framework Programme for Research and Innovation (2014-2020) (n. ${ }^{\circ}$ 731685). Next-Lab extiende el ecosistema Go-Lab a las habilidades del siglo XXI, facilita la autoevaluación, la evaluación por pares y el desarrollo de portafolios, así como el aprendizaje por modelado. Además, Next-Lab hace un esfuerzo especial al fomentar la actitud positiva hacia la ciencia y el área STEM en general, no solo en la enseñanza secundaria, sino también en la enseñanza primaria. Precisamente una de las principales limitaciones del proyecto Go-Lab, además de los problemas de conexión en algunos centros educativos, era la dificultad conceptual de algunos laboratorios para adaptarse a la etapa de educación primaria. Por ello el futuro del proyecto Go-Lab materializado en NextLab se centra en simplificar los laboratorios para que sean aptos también para trabajar con los objetivos del currículo educativo de educación primaria.

\section{Referencias bibliográficas}

Balamuralithara, B. y Woods, P.C. (2009). Virtual laboratories in engineering education: The simulation lab and remote lab. Computer Applications in Engineering Education, 17(1), 108-118.

<https://doi.org/10.1002/cae.20186> 
Bell, R.L. y Trundle, K.C. (2008). The use of a computer simulation to promote scientific conceptions of moon phases. Journal of Research in Science Teaching, 45, 346-372. $<$ https://doi.org/10.1002/tea.20227>

Bybee, R. (ed.) (2002). Learning science and the science of learning. Arlington, VA: NSTA Press.

Chambers, R.; Chuen Yee Lo, B. y Allen, N.B. (2008). The Impact of Intensive Mindfulness Training on Attentional Control, Cognitive Style, and Affect. Cognitive Therapy and Research, 32, 303-322. <https://doi.org/10.1007/s10608-007-9119-0>

Corter, J.E.; Esche, S.K.; Chassapis, C.; Ma, J. y Nickerson, J.V. (2011). Process and learning outcomes from remotely-operated, simulated, and hands-on student laboratories. Computers \& Education, 57(3), 2054-2067.

De Jong, T. (2006). Technological advances in inquiry learning. Science, 312(5773), $532-533$.

De Jong, T.; Linn, M.C. y Zacharia, Z.C. (2013). Physical and virtual laboratories in science and engineering education. Science, 340(6130), 305-308.

Dziabenko, O. y BudnyK, O. (2019). Go-Lab ecosystem: Using online laboratories in a primary school, EDULEARN19 Proceedings. EDULEARN19 (11th annual International Conference on Education and New Learning Technologies). Palma de Mallorca.

EACEA P9 Eurydice (2011). Science education in Europe: National policies, practices and research. Bruselas: Education, Audiovisual and Culture Executive Agency (EACEA) P9 Eurydice. Recuperado de <https://op.europa.eu/en/publication detail/-/publication/bae53054-c26c-4c9f-8366-5f95e2187634>.

Eizagirre Sagardia, A.I.; Altuna Urdin, J. y Fernández Fernández, I.B. (2018). Los entornos activo-colaborativos de aprendizaje como buenas prácticas en el desarrollo de competencias transversales en la formación profesional de la Comunidad Autónoma del País Vasco. Educar, 54(2), 331-349. $<$ https://doi.org/10.5565/rev/educar.880>

Feisel, L.D. y Rosa, A.J. (2005). The Role of the Laboratory in Undergraduate Engineering Education. Journal of Engineering Education, 94(1), 121-130. <https://doi.org/10.1002/j.2168-9830.2005.tb00833.x>

Finkelstein, N.D.; Adams, W.K.; Keller, C.J.; Kohl, P.B.; Perkins, K.K.; PodoLefsky, N.S.; Reid, S. y LeMaster, R. (2005). When learning about the real world is better done virtually: A study of substituting computer simulations for laboratory equipment. Physical Review Special Topics: Physics Education Research, 1(1), 010103-1.

Hofstein, A. y LunetTa, V.N. (2004). The laboratory in science education: Foundations for the twenty-first century. Science Education, 88(1), 28-54. <https://doi.org/10.1002/sce.10106>

Huppert, A.; Blasius, B. y Stone, L. (2002). A model of phytoplankton blooms. American Naturalist, 159, 156-171.

Ma, J. y Nickerson, J.V. (2006). Hands-On, Simulated, and Remote Laboratories: A Comparative Literature Review. ACM Computing Surveys, 38(3), 1-24.

Minner, D.D.; Levy, A.J. y Century, J. (2010). Inquiry-based science instructionwhat is it and does it matter?: Results from a research synthesis years 1984 to 2002. Journal of Research in Science Teaching, 47(4), 474-496.

<https://doi.org/10.1002/tea.20347> 
National Coalition for Literacy (2010, November 12). National Education Technology Plan 2010: Transforming American Education: Learning Powered by Technology. Recuperado de <https://library.educause.edu/resources/2010/11/nationaleducation-technology-plan-2010-transforming-american-education-learningpowered-by-technology>

Orduña, P. et al. (2018). The WebLab-Deusto Remote Laboratory Management System Architecture: Achieving Scalability, Interoperability, and Federation of Remote Experimentation. En M. Auer; A. AzAD; A. EdwardS y T. DE Jong (eds.). Cyber-Physical Laboratories in Engineering and Science Education (pp. 17-42). Cham: Springer.

Paavola, S. y Hakkarainen, K. (2018). Community of inquiry and inquiry-based learning. Encyclopedia of Educational Philosophy and Theory. Singapur: Springer.

Pedaste, M.; Mäeots, M.; SiIman, L.A.; De Jong, T.; Van Riesen, S.A.; Kamp, E.T. y TsourLIDAKI, E. (2015). Phases of inquiry-based learning: Definitions and the inquiry cycle. Educational Research Review, 14, 47-61.

Rocard, M.; Csermely, P.; Jorde, D.; Lenzen, D.; Walberg-Henrikson, H. y Немmo, V. (2007). Science education now: A renewed pedagogy for the future of Europe. Bruselas: European Commission. Directorate-General for Research. Recuperado de <http://ec.europa.eu/research/science-society/document_library/ pdf_06/report-rocard-on-science-education_en.pdf $>$.

Zacharia, Z.C. y Olympiou, G. (2011). Physical versus virtual manipulative experimentation in physics learning. Learning and Instruction, 21(3), 317-331. 